\title{
La felicidad en tiempos de pandemia y educación virtual: un estudio en universitarios del Altiplano
}

\author{
Happiness in times of pandemic and virtual education: a study in \\ university students from the Altiplano
}

\author{
Kleiber Rosendo Vargas Pacosonco ${ }^{\mathrm{a}}$ \\ https://orcid.org/0000-0002-4481-188X \\ Zaida Esther Callata Gallegos ${ }^{\text {b }}$ \\ https://orcid.org/0000-0001-8242-2511 \\ Universidad Nacional del Altiplano, Puno, Perú. \\ a kvargas@unap.edu.pe \\ b zaidacallata@unap.edu.pe
}

Recibido: 06/02/2021

Aceptado: 20/05/2021

Pub7icado: 15/06/2021

\section{Resumen}

El artículo consideró como objetivo identificar el nivel de felicidad en estudiantes universitarios que atraviesan una coyuntura atípica ocasionada por la pandemia Covid-19, la misma que tuvo como una de sus consecuencias en el ámbito educativo el desarrollo de clases a partir de sistemas remotos y virtuales, sucesos nuevos en un país como el Perú, los cuales después de un periodo de implementación registran efectos peculiares que permiten su estudio, la investigación utilizó el método no experimental, de corte transversal, tipo descriptivo de carácter cuantitativo en una muestra estratificada y proporcional de 224 universitarios de la Facultad de Ciencias de la Educación de la UNA - Puno, la única variable de estudio se evaluó con la Escala de Felicidad de Lima que consta de 27 reactivos. Entre los resultados más resaltantes se tiene que el $33.9 \%$, percibe la felicidad en un nivel bajo, el $38.8 \%$ en un nivel medio y un $27.2 \%$ en un nivel alto. Se concluye el estudio afirmando que el nivel de felicidad de los estudiantes no responde a niveles deseados, debido a situaciones nulas de socialización académica causada por la emergencia sanitaria.

Palabras clave: Covid-19; educación virtual; estudiantes; felicidad.

\begin{abstract}
The article considered the objective of identifying the level of happiness in university students who are going through an atypical situation caused by the Covid-19 pandemic, the same one that had as one of its consequences in the educational field the development of classes from remote and virtual systems, new events in a country like Peru, which after a period of their implementation register peculiar effects that allow their study, the research used the non-experimental method, cross section, descriptive type of quantitative nature in a stratified and proportional sample of 224 university students from the Faculty of Education Sciences of the UNA - Puno, The only study variable was evaluated with the Lima Happiness Scale, which consists of 27 items. Among the most outstanding results we have that $33.9 \%$, perceives happiness at a low level, $38.8 \%$ at a medium level and $27.2 \%$ at a high level. The study concludes stating that the level of happiness of the students does not respond to desired levels, due to null situations of academic socialization caused by the health emergency.
\end{abstract}

Key words: Covid-19; virtual education; students; happiness. 


\section{Introducción}

El ser humano tiende a buscar la felicidad como objetivo supremo, sin embargo es muy frecuente encontrar a personas sumidas en la tristeza, desesperación, pesimismo y desinterés, vivir en un mundo globalizado propicia que uno de los problemas de nuestro tiempo sea que las personas se encuentren atrapadas con una sensación de falta de sentido y ganas de vivir (Eugenio, Huansi y Flores, 2015). Los estudiantes universitarios no son ajenos a esta realidad más aun en tiempos de pandemia y educación virtual a los cuales se han tenido que adaptar.

Identificar el nivel de felicidad en universitarios es relevante puesto que permite conocer las causas asociadas a ella, debido a que influirán en el cumplimiento de las metas y la satisfacción de las expectativas personales. La felicidad es un estado de ánimo que se ocasiona en la persona cuando cree haber logrado una meta buena (Eugenio et al., 2015). Sin embargo la pandemia Covid-19 alteró la manera de habitar el mundo, haciendo de los espacios públicos escenarios de amenaza para la vida y dando relevancia y prioridad al espacio privado como lugar aparentemente seguro y sagrado donde se vive con cierta protección y seguridad (Amparo et al., 2020), lo cual causó que la educación se realice de manera virtual y pudo haber generado cambios en la percepción de felicidad.

112 Definir el término Felicidad nos lleva a conclusiones antropológicas y filosóficas tan diversas como disímiles, muchos filósofos han buscado analizar esta acepción desde lo más profundo del ser y el pensamiento originando un $\sin$ fin de interpretaciones dando lugar a corrientes filosóficas (Andía, 2015) marcadas desde un sentido muy humano que busca una realización espiritual y somática (Zelenski et al., 2008). Aristóteles sostiene que "la felicidad es como un bien supremo, es aquello que da sentido y finalidad a otro fin querido por el hombre" (Aristóteles, 1985), el cual depende del desarrollo de la inteligencia emocional (Millán et al., 2020). Posterior a ello, durante la época helenística, surgió la ataraxia como un camino alternativo a la felicidad, ya que no todos estaban llamados a vivir una vida en virtud (Leahey, 2011). Es así que se pueden distinguir dos concepciones de felicidad, una concebida como eudaimonía que proviene de la sabiduría, y otra hedónica, orientada a la posesión de bienes y por ende, la satisfacción de necesidades (Arias et al., 2016), debido a que el ser humano busca ser feliz en cada momento de su vida (Moccia, 2016).

En el contexto de la educación superior, la felicidad denominada también bienestar subjetivo (Ramírez, 2013), debería tener por finalidad última responder a la promoción del bienestar personal y social (Bisquerra y Hernández, 2017). Es de interés para las áreas concernientes al bienestar universitario que sus estudiantes satisfagan diversas necesidades que le permitan una realización personal plena, es decir que la felicidad se alcance por la realización del propio potencial (Davey, 2017). La percepción que se tiene de sus recursos emocionales favorece en su desempeño académico y futuro profesional, por este motivo existe una variabilidad conceptual, sobre las causas que generan e incrementan la felicidad personal y las orientaciones que las estudian (Gutiérrez et al., 2013).

En la etapa académica el bienestar (equivalente al sentimiento de felicidad) debería ser uno de los objetivos de la educación, lo cual repercutiría en el profesorado, alumnado, familia y por extensión a la sociedad en general (Bisquerra y Hernández, 2017). El bienestar tiene una dimensión personal (se le brinda mayor prioridad) y otra social. La felicidad muchas veces se busca por caminos equivocados, que pueden conducir a comportamientos de riesgo, como consumo de drogas (Bisquerra y Hernández, 2017), más aún en la etapa universitaria estos riesgos acarrean deserción estudiantil, alcoholismo, embarazos no planificados, entre otros, por lo que conviene desarrollar fortalezas personales y un estado de calma que ayude a alcanzar satisfacción en la vida.

Diversos estudios demuestran que los jóvenes universitarios se sienten felices (Millán etal., 2020), pero que este sentimiento responde a diversos factores que se interrelacionan para generar el estado de bienestar que permita a los jóvenes tranquilidad en el transcurso de su vida académica y social. Ellos perciben la felicidad de acuerdo a factores como: tiempo, forma de alcanzarla y momentos memorables. Los estudiantes de la educación superior universitaria creen que se trata de un estado de la mente o un sentimiento, sin embargo lo que les causa real felicidad es estar en compañía de los seres amados (padres, hermanos, amigos, pareja sentimental) hecho que no depende de factores externos (Mercado, 2014), sino que se 
centra en factores internos como el sentimiento de cariño, aceptación, autoestima y valoración frente al entorno familiar, académico y social. A pesar de la poca comprensión que brinda el sentimiento de la felicidad, los universitarios saben hacia dónde van y trazan metas, confían en su capacidad para producir bienestar (Zubieta, Delfino y Gisela, 2010).

Existen diversas condiciones que impiden la felicidad del estudiante universitario, tales como: el bajo nivel de conocimiento del contenido de las asignaturas (Guadalupe et al., 2017), un sistema educativo que no considera la felicidad como una variable de gestión educativa (Concepción et al., 2018), el mal uso de las redes sociales y la dedicación a la obtención de dinero que sería un factor conducente a la felicidad (Gabini, 2017). Los estudiantes prefieren la comunicación por las redes sociales frente al diálogo en persona, el ocio pasivo frente al activo, en definitiva el ocio sedentario (Navarro, Martinez y Pérez, 2017), que puede traer consigo la soledad académica (Vargas et al., 2021).

Los estudiantes universitarios tienen como objeto de vida la conclusión de una carrera profesional que les permita realizarse como profesionales en la comunidad, por ello el aspecto académico y el futuro laboral exitoso son una vía en el camino de la felicidad, razón por la que en diversos estudios sobre felicidad se ha encontrado que la autoeficacia, el engagement, la satisfacción y el estrés académico (Salanova et al., 2005), se relacionan con el rendimiento académico (Arias et al., 2016). Además es necesario comprender que el aspecto académico y laboral no se caracterizan sólo por tener sentimientos placenteros o experiencias afectivas positivas, sino que involucran la necesidad que esta esfera vital sea significativa para el ser humano (Salas, Alegre y Fernández, 2013), esto ocurre porque la felicidad que se busca también se adecúa al contexto y la persona.

La medición de la felicidad es un tema en boga a nivel internacional (Beytía y Calvo, 2013), motivo por el cual en el 2016 se utilizó la Escala de Felicidad de Lima (EFL), que presenta adecuadas propiedades psicométricas (Gallegos et al., 2016), que permiten recomendar su empleo como instrumento de evaluación de la felicidad en poblaciones universitarias (Arias et al., 2016), pues es un instrumento que posee características fáciles para su administración (DeVellis, 2016), y que tiene un número reducido de ítems (Gabini, 2017). Un objetivo adicional del presente escrito es identificar la diferencia en el nivel de felicidad entre hombres y mujeres (Toribio et al., 2021), ya que existen diferencias significativas de género, los varones por ejemplo, hacen más ejercicio físico y las mujeres se concentran en prácticas religiosas (Gallegos et al., 2016). Es importante señalar que factores como la religión contribuyen al bienestar personal (Concepción et al., 2018), lo mismo que el aspecto corporal contribuye a la construcción de la identidad (Delgado et al., 2018).

El valor del presente estudio radica en que la felicidad ha sido asociada positivamente al éxito académico, porque favorece el afrontamiento de situaciones estresantes de la vida universitaria; además tiene efectos positivos en la salud y el bienestar personal y social, que contribuyen en la mejora de la calidad de vida (Caballero y Sánchez, 2018), hecho que permite mencionar que cada uno de los resultados presentados contribuirán en gran dimensión en el desarrollo humano y profesional de los estudiantes universitarios, puesto que se ha comprobado que a mayor felicidad se tienen más probabilidades de finalizar los estudios universitarios (Caballero y Sánchez, 2018).

\section{Materiales y métodos}

El periodo de investigación corresponde al primer semestre del año 2020 (junio a noviembre) en un contexto de enseñanza virtual a causa de la pandemia Covid-19. La investigación fue no experimental, de corte transversal, tipo descriptivo y de carácter cuantitativo; se buscó identificar el estado de la variable felicidad en un determinado momento en estudiantes universitarios puneños (Hernández \& Mendoza, 2018).

La población estuvo conformada por 2265 alumnos matriculados en el semestre académico 2020 - I, de la Facultad de Ciencias de la Educación de la Universidad Nacional del Altiplano - Puno, el tipo de muestreo fue estratificado y proporcional, seleccionando aleatoriamente a 224 estudiantes de las carreras de Educación Primaria (65 estudiantes), Educación Inicial (52 estudiantes), Educación Física (44 estudiantes) y Educación Secundaria (63 estudiantes), de acuerdo al sexo se tiene: el $21 \%$ de varones y el $79 \%$ de mujeres, los mismos que bordeaban la edad de 16 a 20 años (47.3\%), de 21 a 25 años (49.6\%) y de 26 años a más $(3.1 \%)$. 


\section{Instrumento}

Alarcón (2006), menciona que el instrumento consta de 27 reactivos agrupados en cuatro factores, el primero (sentido positivo de la vida) agrupa 11 ítems, asociados a "la percepción y vivencia de la libertad; visión positiva de la vida y del futuro" (García, Gallego y Pérez, 2009), el segundo (satisfacción con la vida) lo integran 6 ítems, entendidos como "el juicio que posee una persona acerca de su propia vida" (Vera, Velasco, Grubits y Salazar, 2016), el tercero (realización personal) suma también 06 ítems, que expresan "plenitud de felicidad permanente, equilibrio emocional" (Davey, 2017), y el último factor (alegría de vivir) reúne 04 ítems, comprendidos en el concepto de "plenitud en la vida", todos los reactivos responden a una escala tipo Likert que va desde "totalmente de acuerdo" a "totalmente en desacuerdo".

Previo a la aplicación del instrumento, se dialogó con los docentes, con el objetivo de lograr su apoyo en el proceso investigativo desarrollado frente a la medición del nivel de felicidad. Después de obtener la autorización de los docentes responsables de las asignaturas, los cuestionarios virtuales (formulario google) fueron aplicados mediante el uso del correo institucional de cada estudiante durante los meses de setiembre y octubre de 2020, la participación fue voluntaria, garantizándose la privacidad y el secreto de todos los datos, siendo responsables en todo momento de los preceptos de ética y conducta profesional. El examen de datos fue realizado mediante el paquete estadístico SPSS (versión 22.0), donde se obtuvo los estadísticos descriptivos de la Media (=97.290) y la Desviación (=19.452), además se calculó el estadístico de fiabilidad Alfa de Cronbach $(=0.952)$.

\section{Resultados}

En respuesta al objetivo principal se encontró que el nivel de felicidad de los estudiantes de la Facultad de Ciencias de la Educación de la Universidad Nacional del Altiplano Puno en el año 2020 , responde a un nivel bajo en un $33.9 \%$, un nivel medio al $38.8 \%$ y un nivel alto a $27.2 \%$, para mayor detalle los resultados obtenidos se presentan en cuatro factores detallados a continuación:
18. Tengo problemas tan hondos que me quitan la tranquilidad. 224 respuestas

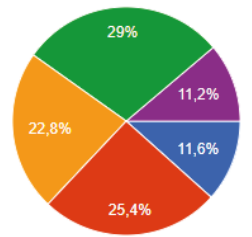

De acuerdo

toni en desacuerdo - En desacuerdo Totalmente en desacuerdo

Figura 1. Sentido Positivo de la Vida (Respuesta ítem 18) Factor 1.

Fuente: Datos registrados mediante el formulario google aplicado a los estudiantes de la Facultad de Ciencias de la Educación de la UNA - Puno, año 2020 .

En la figura 1, del 100\% (224) de respuestas, un total del $40.2 \%$ rechaza la afirmación, mientras que un global del 59.8\% la aceptan, lo que indica que 134 estudiantes evidencian rasgos de desánimo, derrota, angustia, desesperanza o vacío existencial, hecho que afectará de sobremanera su desempeño académico y frustrará el logro de sus metas profesionales. Las respuestas afirmativas de los universitarios en el factor 1, también demuestran características que reflejan que éstos estudiantes sienten que la vida ha sido injusta con ellos y sienten que nunca podrán ser felices.

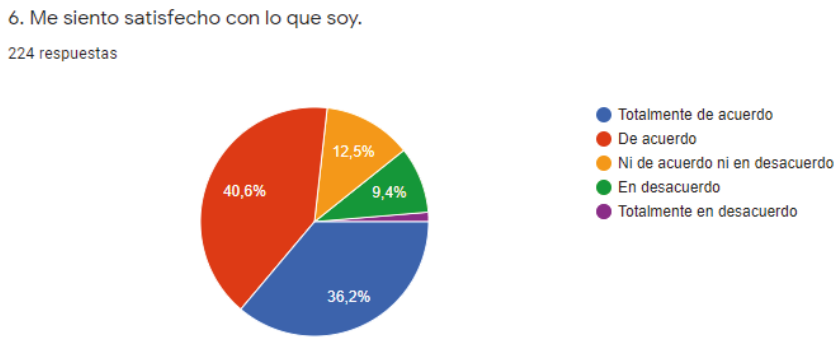

Figura 2. Satisfacción con la Vida (Respuesta ítem 6) Factor 2.

Fuente: Datos registrados mediante el formulario google aplicado a los estudiantes de la Facultad de Ciencias de la Educación de la UNA - Puno, año 2020 .

En la figura 2, el 76.8\% (172 estudiantes) de las respuestas es positiva frente a la afirmación, lo que indica que los alumnos sienten que la vida ha sido buena con ellos, que sus condiciones son excelentes y que en la mayoría de las cosas, la vida está cerca del ideal que esperaron, sin embargo, la respuesta negativa del $23.2 \%$ (52 estudiantes) muestra indicios que este grupo de alumnos universitarios no se encuentran satisfechos por lo que han logrado y creen que no están donde tienen que estar, o se encuentran a una distancia remota del ideal de su vida, situaciones que influyen en su desarrollo personal y social. 
9. Si volviese a nacer, no cambiaria casi nada en mi vida. 224 respuestas
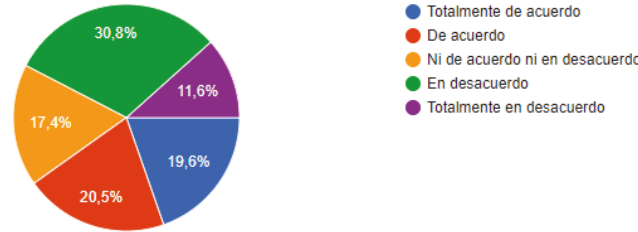

Totalmente en desacuerdo

Figura 3. Realización Personal (Respuesta ítem 9) - Factor 3.

Fuente: Datos registrados mediante el formulario google aplicado a los estudiantes de la Facultad de Ciencias de la Educación de la UNA - Puno, año 2020 .

En la figura 3, la respuesta afirmativa a este ítem señala autosuficiencia, calma emocional, apacibilidad o alguna condición que permita conseguir el estado de felicidad, lo que se puede observar en un total de 90 estudiantes (40.1\%) que se caracterizan por sentirse personas realizadas que han conseguido las cosas que son importantes en la vida, no obstante, un conjunto de 134 universitarios (59.8\%) muestran síntomas de dependencia, subyugación, miedo o estados similares que no les permiten sentir felicidad, hecho que afectará en su performance profesional.

12. Es maravilloso vivir.

224 respuestas
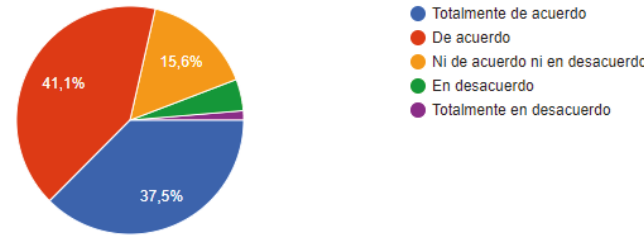

- En desacuerdo

Totalmente en desacuerdo

Figura 4. Alegría de vivir (Respuesta ítem 12) - Factor 4.

Fuente: Datos registrados mediante el formulario google aplicado a los estudiantes de la Facultad de Ciencias de la Educación de la UNA - Puno, año 2020.

La figura 4, evidencia que 48 estudiantes (21.4\%) no sienten lo extraordinario de vivir, puesto que refieren experiencias negativas de la vida y se sienten generalmente mal, ellos necesitan aceptar que la vida está llena de experiencias positivas que pueden contribuir en su existencia, al igual que 176 estudiantes $(78.6 \%)$ que perciben una vida con cualidades cercanas a la felicidad, ya que se caracterizan por ser personas optimistas que han experimentado la alegría de vivir.

Los efectos mostrados en las figuras 1, 2, 3 y 4 son el resultado de la etapa de crisis sanitaria que se vive a nivel mundial, hecho que causó la virtualización de la educación en un $100 \%$, aspecto que influyó de manera decisiva en los estudiantes, y se detalla en la figura 5:
$\mathrm{H}$. Las clases virtuales me hacen sentir:

224 respuestas

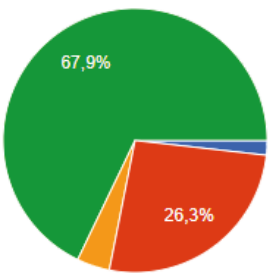

Alegre

Tranquila (0)

Triste

Estresada (0)

Figura 5. Sensación que provoca las clases virtuales en los estudiantes universitarios.

Fuente: Datos registrados mediante el formulario google aplicado a los estudiantes de la Facultad de Ciencias de la Educación de la UNA - Puno, año 2020 .

En la figura 5, el resultado muestra que el 1.8\% de estudiantes sienten alegría con las clases virtuales, el $26.3 \%$ siente tranquilidad, empero el $4 \%$ siente tristeza y de manera general el $67.9 \%$ siente estrés motivado por la virtualización de la educación. Los datos presentados para graficar la percepción del sentido positivo de la vida (factor 1), la satisfacción con la vida (factor 2), la realización personal (factor 3) y la alegría de vivir (factor 4) se relacionan a la figura 5 , debido a que la etapa de enseñanza virtual influye en los sentimientos de los estudiantes universitarios.

\section{Discusión}

Un resultado valioso que se encontró es el nivel medio de felicidad que responde al $38.8 \%$, dato que es similar al 38\% hallado por Davey (2017), los resultados coinciden puesto que los estudios se realizaron en poblaciones similares, asimismo, en referencia al nivel alto se demuestra que las mujeres $(28.8 \%)$ sienten un nivel mayor en comparación con los varones $(21.3 \%)$, esta cifra se relaciona con el resultado de Santoya, Garcés y Tezón (2018) en donde, las mujeres presentaron promedios superiores a los hombres, lo que confirma la validez del trabajo. Además, el índice de felicidad encontrado no supera el $50 \%$, hecho que ratifica que las evocaciones que vivimos diariamente responden en su mayoría a sensaciones de tristeza e intranquilidad que son un común denominador en diversas investigaciones a nivel internacional.

Al identificar los datos sobre el sentido positivo de la vida (véase la figura 1), se halló que el $40.2 \%$ rechaza la afirmación del reactivo 18 "tengo problemas tan hondos que me quitan la tranquilidad", por ello Gamero, Medina y Escobar (2019) explican que este nivel de rechazo 
responde a que los años de educación reducen en un $1 \%$ la probabilidad de encontrarse en un nivel insatisfecho de felicidad, resultados análogos que revalidan la importancia de los logros académicos en beneficio de la felicidad. Ahora no se puede dejar de lado al 59.8\% de estudiantes que aceptan la aseveración del ítem 18, lo que indica que este grupo de universitarios, a pesar de seguir estudios superiores evidencian desánimo, derrota, angustia, desesperanza o vacío existencial, rasgos que se relacionan con la nula socialización académica que los estudiantes vivieron en la etapa de educación virtual.

La relación relevante entre la variable felicidad como factor que impacta en el rendimiento académico encontrada por Ravina, Ahumada y Gálvez (2019), se legitima con los porcentajes encontrados en referencia a las ubicaciones de los estudiantes en el ranking académico, pues el $33.0 \%$ se ubican en el medio superior, el $35.7 \%$ en el tercio superior, el $12.1 \%$ en el quinto superior y el $10.7 \%$ décimo superior, situación de coincidencia que permite reflexionar sobre el efecto positivo de los sentimientos en la formación profesional. Asimismo, un total del $76.8 \%$ de alumnos brinda una respuesta positiva a la afirmación "me siento satisfecho con lo que soy" (véase la figura 2), lo que expresa satisfacción por lo que han logrado $\mathrm{y}$ se relaciona con el rendimiento académico aceptable en el proceso de formación académica.

Los resultados del factor "Realización Personal" en referencia al ítem 9 "si volviese a nacer, no cambiaría casi nada en mi vida" (véase figura 3) muestran que los universitarios perciben un nivel promedio de felicidad, lo que concuerda con Hernández y Carranza (2017) que manifiestan que a mayor felicidad mayores niveles de optimismo. Ahora bien, el 59.8\% de estudiantes perciben sensaciones de sumisión, sometimiento, miedo o algún otro estado que no les permite sentir felicidad, resultado comparable con lo hallado por Fernández (2017) donde $42 \%$ de estudiantes consideran que no han logrado las metas que son importantes para la vida, indicando no tener una felicidad plena, ni haber conseguido autosuficiencia, ni tranquilidad emocional, esta semejanza de datos permite evidenciar los niveles disminuidos de felicidad en los estudiantes de la educación superior.

Fernández (2017), encontró que el nivel de felicidad de los estudiantes en el nivel bajo y muy bajo responde al 14\%, resultado que sostiene la presente investigación, ya que el $21.4 \%$ de universitarios no logra niveles óptimos de felicidad, esto debido a que los alumnos universitarios (también gran parte de la sociedad) se equivocan al definir o delimitar lo que significa la felicidad. La realidad hace ver que la felicidad no significa sonreír en todo momento, sino entender que las dificultades siempre estarán presentes, pero que a pesar de ello la vida se enmarca en una constante búsqueda de la ansiada felicidad, que en realidad nunca podrá ser encontrada de manera plena.

Aún se desconocen los alcances de la pandemia, tanto en términos económicos, políticos, educativos, como en materia de salud mental de los/as adolescentes (Vásquez, 2021), por este motivo el $67.9 \%$ de estudiantes afectados por el estrés (véase figura 5), es un porcentaje que permite ensayar sobre la tendencia de virtualización de la educación que será un problema grave en la salud psíquica (estrés) y física (disminución de la visión) de los alumnos, lo cual debe permitir reflexionar frente a la enseñanza que se aplica mediante un ordenador y las bondades del internet, razón por la que se debe tomar una posición crítico-reflexiva frente a esta situación.

\section{Conclusiones}

El nivel de felicidad de los estudiantes de la Universidad Nacional del Altiplano no responde a niveles deseados, ya que los universitarios no sienten positivamente los niveles de felicidad, estos datos responden a niveles nulos de socialización académica causada por la emergencia sanitaria. Además, en referencia al nivel alto de felicidad las mujeres sienten un nivel mayor en comparación con los varones, circunstancia que determina que las mujeres poseen mayor resistencia a situaciones que puedan provocar sensaciones de tristeza o estrés.

La tristeza es una marca existencial en el ser humano, y los estudiantes no escapan a esta realidad, pues perciben sensaciones de tristeza y estrés, efecto que permite reflexionar que una de las causas responda a que los seres humanos no buscan adecuadamente la felicidad, y que además no se tiene un concepto claro que permita definirla.

Una de las consideraciones que se tiene para sentir la felicidad en la vida responde al logro de distintos objetivos, en este caso estudiar una carrera universitaria, los alumnos expresan satisfacción 
por lo que han alcanzado y ello se relaciona con su rendimiento académico aceptable en el proceso de formación profesional, pues el ideario general de los alumnos recae en la idea de obtener buenas calificaciones que aparentemente les genera ciertos niveles de felicidad.

Las consecuencias ocasionadas por la etapa de emergencia sanitaria y las clases virtuales son preocupantes, ya que los alumnos sienten sensaciones que no les permiten sentir felicidad, además se evidencia que los universitarios necesitan vivenciar que la vida está llena de experiencias positivas, estos resultados refieren la necesidad de intervenciones que contribuyan en la construcción de la felicidad.

La educación presencial no podrá ser superada por la educación virtual. En el contexto de la pandemia la virtualización de la educación fue una necesidad, sin embargo, como todo suceso nuevo y desconocido para algunos países como el nuestro, después de una etapa de experimentación que duró aproximadamente un año (2020), los efectos secundarios ocasionaron que los estudiantes sientan estrés y tristeza a causa de recibir enseñanza a través de la pantalla de un ordenador.

\section{Referencias Bibliográficas}

Alarcón, R. (2006). Desarrollo de una escala factorial para medir la felicidad. Interamerican Journal of Psychology, 40(1), 99-106.

Amparo, P., Palacios, N., \& Morillo, J. P. (2020). Acompañamiento desde una ética de la vida: Para educar en tiempos de pandemia. 11-24.

Andía, V. (2015). Analisis de los Indices de Felicidad como Medida de Bienestar en la Escuela Profesional de Administración UNA- PUNO. Revista de Investigaciones Altoandinas, 17(3), 395-402.

Arias, W.L., Caycho, T., Ventura, J.L., Maquera, C., Ramírez, M., \& Tamayo, X. (2016). Análisis exploratorio de la escala de felicidad de Lima en universitarios de Arequipa. Psychologia. Avances de La Disciplina, 10(1), 11.

Aristóteles. (1985). Ética nicomáquea (E. Gredos, Ed.). Madrid.

Beytía, P., \& Calvo, E. (2013). ¿Cómo Medir La Felicidad? (How to Measure Happiness?). SSRN Electronic Journal, 1-10. https://doi. org/10.2139/ssrn.2302809

Bisquerra Alzina, R., \& Hernández Paniello, S. (2017). Positive psychology, emotional education and the happy classrooms program. Papeles Del Psicologo, 38(1), 58-65. https:// doi.org/10.23923/pap.psicol2017.2822

Caballero García, P. Á., \& Sánchez Ruiz, S. (2018). La felicidad en estudiantes universitarios. ¿Existen diferencias según género, edad o elección de estudios? Revista Electrónica Interuniversitaria de Formación Del Profesorado, 21(3), 1-18. https://doi. org/10.6018/reifop.21.3.336721

Concepción, L. A., Dextre, C. E., Landauro, E. D. R., \& Vásquez, A. R. (2018). Medición de la Felicidad en Profesores de Dos Centros Educativos de Lima (PONTIFICIA UNIVERSIDAD CATÓLICA DEL PERÚ). Retrieved from http://tesis.pucp.edu.pe/repositorio/ bitstream/handle/20.500.12404/13187/ C O N C E P C I O N D E X T R E F E L I C I D A D E D U C A T I V O S. pdf? sequence $=1 \&$ is $\bar{A}$ llowed $=\mathrm{y}$

Davey Contreras, K. A. (2017). Engagement and Happiness levels in Lima's working college students. Revista de Investigación En Psicología, 20, 389-406. https://doi.org/ https://doi.org/10.15381/rinvp.v20i2.14049

Delgado, P., Caamaño, F., Martínez, C., Jerez, D., Carter, B., García, F., \& Latorre, P. (2018). La obesidad infantil y su asociación con el sentimiento de infelicidad y bajos niveles de autoestima en niños de centros educativos públicos. Nutrición Hospitalaria, 35, 5. https://doi.org/http://dx.doi.org/10.20960/ nh.1424

DeVellis, R. F. (2016). Scale development: Theory and applications (4th edició; S. Publications, Ed.). California.

Eugenio Rosali, Ú., Huansi, L., \& Flores Esteba, I. E. (2015). Felicidad: un estudio comparativo en estudiantes universitarios de Psicología y Administración de UNA. 21-29.

Fernández Ferro, C. O. (2017). Niveles de felicidad en estudiantes del Instituto de Educación Superior Daniel Alcides Carrión, 2017 (UNIVERSIDAD INCA GARCILASO DE LA VEGA; Vol. 6). Retrieved from http://repositorio.unan. edu.ni/2986/1/5624.pdf\%0Ahttp://fiskal. kemenkeu.go.id/ejournal\%0Ahttp://dx.doi. org/10.1016/j.cirp.2016.06.001\%0Ahttp:// dx.doi.org/10.1016/j.powtec.2016.12. $055 \% 0 \mathrm{Ahttps} / / / \mathrm{doi}$.org/10.1016/j. ijfatigue.2019.02.006\%0Ahttps://doi. org/10.1 
Gabini, S. (2017). Adaptación Y Validación De Un Instrumento Para Medir Felicidad En El Trabajo. Revista Interamericana de Psicología Ocupacional, 36(2), 36-45. https://doi.org/10.21772/ripo.v36n2a03

Gallegos, A., Walter, L., Rodríguez, C., León, V., Luís, J., Fernández, M., ... Butilier, T. (2016). de la escala de felicidad de L ima. 13-24.

Gamero Tafur, K. E., Medina Martínez, E. M., \& Escobar Espinoza, Á. A. (2019). LA FELICIDAD EN ESTUDIANTES UNIVERSITARIOS DE CIENCIAS ECONÓMICAS: ALGUNOS DETERMINANTES SOCIOECONÓMICOS EN LA CIUDAD DE CARTAGENA DE INDIAS. Kemampuan Koneksi Matematis (Tinjauan Terhadap Pendekatan Pembelajaran Savi), 53(9), 1689-1699. https://doi.org/10.22519/22157360.1032

García-Alandete, J., Gallego-Pérez, J. F., \& Pérez-Delgado, E. (2009). Sentido de la vida y desesperanza: Un estudio empírico. Universitas Psychologica, 8(2), 447-454.

Guadalupe, C., León, J., Rodríguez, J., \& Vargas, S. (2017). ESTADO DE LA EDUCACIÓN EN EL PERU: Análisis y perspectivas de la educación básica (Primera ed; I. y E. A. E.I.R.L., Ed.). Retrieved from http://www.grade.org.pe/ forge/descargas/Estadodelaeducación en el Perú.pdf

Gutiérrez, J. L. P., Aragón, S. R., Lagunes, I. R., \& López Parra, M. S. (2013). Escala de Felicidad en la Pareja: Desarrollo y Validación. Acta de Investigación Psicológica, 3(3), 1280-1297. https://doi.org/https://doi.org/10.1016/s20074719(13)70967-0

Hernández-Sampieri, R., \& Mendoza, C. (2018). Metodología de la investigación. Las rutas cuantitativa, cualitativa y mixta. In Highered. Mheducation.Com (McGRAW-HIL). México.

Hernández, R. M., \& Carranza Esteban, R.F.(2017). Felicidad, optimismo y autorrealización en estudiantes de un programa de educación superior para adultos. Interdisciplinaria: Revista de Psicología y Ciencias Afines, 34(2). https://doi.org/10.16888/interd.2017.34.2.5

Leahey, T. H. (2011). Historia de la psicología (Pearson, Ed.). México.

Mercado, M. (2014). Concepto de felicidad en jóvenes. Ajayu, 12(1), 64-78.

Millán-Franco, M., Orgambídez Ramos, A., Domínguez de la Rosa, L., \& Martínez, S. L. (2020). Emotional intelligence and subjective happiness in social work students.
Alternativas, (27), 117-132. https://doi. org/10.14198/ALTERN2020.27.06

Moccia, S.(2016). FELICIDADENELTRABAJO. Junio, 37, 10. Retrieved from https://www. redalyc.org/pdf/2090/209014642004.pdf

Navarro, D., Martinez, R., \& Pérez, I. J. (2017). El enigma de las 3 efes: Fortaleza, fidelidad y felicidad. REVISTA ESPAÑOLA DE EDUCACIÓN FÍSICA Y DEPORTES, (419), 12.

Ramírez, P. E. (2013). Felicidad y Rendimiento Académico: Efecto Moderador de la Felicidad sobre Indicadores de Selección y Rendimiento Académico de Alumnos de Ingeniería Comercial Happiness and Academic Performance. Moderating Effect of Happiness on Selection Indicators And, 6(3), 21-30. https://doi.org/https://doi. org/10.4067/S0718-50062013000300004

Ravina-Ripoll, R., Ahumada-Tello, E., \& GálvezAlbarracín, E. J. (2019). HAPPINESS AS A PREDICTOR OF ACADEMIC PERFORMANCE IN UNIVERSITY STUDENTS . A COMPARATIVE ANALYSIS BETWEEN MEXICO AND SPAIN. CAURIENSIA, XIV, 407-426. https:// doi.org/https://doi.org/10.17398/23404256.14.407

Salanova, M., Martínez, I. M., Bresó, E., Llorens, S., \& Grau, R. (2005). Bienestar psicológico en estudiantes universitarios: facilitadores y obstaculizadores del desempeño académico. Anales de Psicología, 21(1), 10.

Salas, A., Alegre, J., \& Fernández, R. (2013). La medición de la felicidad en el trabajo y sus antecedentes: Un estudio empírico en el área de alergología de los hospitales públicos españoles. Trabajo, 28, 33.

Santoya Montes, Y., Garcés Prettel, M., \& Tezón Boutureira, M. (2018). Las emociones en la vida universitaria: análisis de la relación entre autoconocimiento emocional $y$ autorregulación emocional en adolescentes y jóvenes universitarios. Psicogente, 21(40), 422-439. https://doi.org/10.17081/ psico.21.40.3081

Toribio Pérez, L., González, A., López Fuentes, N. I., Valdez Medina, J. L., González Escobar, S., \& Barneveld, H. O. (2021). Validación de la Escala de Felicidad de Alarcón para adolescentes mexicanos. Psicología Iberoamericana, 20(1), 71-79.

Vargas, K., Yana, M., Pérez, K., Chura, W., \& Alanoca, R. (2021). Aprendizaje colaborativo: una estrategia que humaniza la educación. 
Revista Innova Educación, 3(1), 6-19. https://doi.org/https://doi.org/10.35622/j. $\underline{\text { rie.2020.02.009 }}$

Vásquez-Villegas, M. (2021). Acompañamiento socioemocional en pandemia: tutorías PACE a estudiantes de cuarto año medio. REVISTA SABERES EDUCATIVOS, 6(c), 95-115.

Vera Noriega, J. Á., Velasco Arellanes, F. J., Grubits Goncalves de Oliveira Freire, H. B., \& Salazar Millán, I. G. (2016). Parámetros de inclusión y exclusión en los extremos de la felicidad y satisfacción con la vida en
México. Acta de Investigación Psicológica, 6(2), 2395-2403. https://doi.org/10.1016/j. aipprr.2016.06.002

Zelenski, J., Murphy, S., \& Jenkins, D. (2008). The happyproductive worker thesis revisited. Journal of Happiness Studies, 9, 16.

Zubieta, Delfino, \& Gisela. (2010). Satisfacción con la vida, bienestar psicológico y bienestar social en estudiantes universitarios de Buenos Aires Satisfaction with life, psychological and social well being in college students. Anuario de Investigaciones, 17(1), 277-283. 